\title{
Mechanism of magnetism in stacked nanographite: Theoretical study
}

\author{
Kikuo Harigaya周 \\ Physical Science Division, Electrotechnical Laboratory, \\ Umezono 1-1-4, Tsukuba 305-8568, Japan! \\ National Institute of Materials and Chemical Research, \\ Higashi 1-1, Tsukuba 305-8565, Japan \\ Kanazawa Institute of Technology, \\ Ohgigaoka 7-1, Nonoichi 921-8501, Japan
}

\begin{abstract}
Nanographite systems, where graphene sheets of the orders of the nanometer size are stacked, show novel magnetic properties, such as, spin-glass like behaviors and the change of ESR line widths in the course of gas adsorptions. We theoretically investigate stacking effects in the zigzag nanographite sheets by using a tight binding model with the Hubbard-like onsite interactions. We find a remarkable difference in the magnetic properties between the simple A-A and A-B type stackings. For the simple stacking, there are not magnetic solutions. For the A-B stacking, we find antiferromagnetic solutions for strong onsite repulsions. The local magnetic moments tend to exist at the edge sites in each layer due to the large amplitude of wavefunctions at these sites. Relations with experiments are discussed.
\end{abstract}

PACS numbers: 75.30.-m, 75.70.Cn, 75.10.Lp, 75.40.Mg

*E-mail address: harigaya@etl.go.jp; URL: http://www.etl.go.jp/ ${ }^{*}$ harigaya/; Address after April 2001: National Institute of Advanced Industrial Science and Technology (AIST), Tsukuba 305, Japan

${ }^{\dagger}$ Corresponding address 


\section{Introduction}

Nanographite systems, where graphene sheets of the orders of the nanometer size are stacked, show novel magnetic properties, such as, spin-glass like behaviors [1], and the change of ESR line widths while gas adsorptions [2]. Recently, it has been found [3] that magnetic interactions increase with the decrease of the interlayer distance while water molecules are attached physically. Here, the change of the inter-layer interactions has been anticipated experimentally, but theoretical studies have not been reported yet.

In this paper, we theoretically consider the stacking effects in the zigzag nanographite sheets [4-6] by using a tight binding model with the Hubbard-like onsite interactions $U$. In the papers [4-6], the one dimensional graphite ribbons have been investigated. In this paper, we assume that each graphite sheet has a hexagonal shape with zigzag edges. Such the shape geometry has been used in the semi-empirical study of fluorine doped graphite nanoclusters [7], too. The two stacking types, namely the A-A and A-B types, shown in Fig. 1 are considered in the model. We assume small interlayer interactions $t_{1}$ where two carbon atoms are adjacent between neighboring layers. The circles in Fig. 1 (a) (namely, nanographite a) and Fig. 1 (c) (nanographite $\mathbf{c}$ ) show the sites with the interaction $t_{1}$, and the interaction $t_{1}$ is considered at all the sites in Fig. 1 (b) (nanographite b). Such the interactions preserve the beautiful bipartite property seen in the single hexagonal layer.

The main finding of this paper is a remarkable difference in the magnetic properties between the simple A-A and A-B stackings. For the simple stacking, we have not found magnetic solutions, because the presence of local magnetic moments is suppressed at carbons. For the AB stacking, we have found antiferromagnetic solutions for $U>2 t, t$ being the hopping integral in a layer. The local magnetic moments tend to exist at the edge sites in each layer due to the large amplitude of wavefunctions at these sites. Relations with experiments are discussed extensively.

In Sec II, we explain our model. Sections III and IV are devoted to the total magnetic 
moment per layer, and the local magnetic polarization per site, respectively. In Sec. V, we discuss the local density of states at the edge carbon atoms. This paper is closed with summary in Sec. VI.

\section{Model}

We study the following model with hopping integrals between orbitals of carbon atoms and onsite strong repulsions of the Hubbard type:

$$
\begin{aligned}
H & =-t \sum_{\langle i, j\rangle: \text { intralayer }} \sum_{\sigma}\left(c_{i, \sigma}^{\dagger} c_{j, \sigma}+\text { h.c. }\right) \\
& -t_{1} \sum_{\langle i, j\rangle: \text { interlayer }} \sum_{\sigma}\left(c_{i, \sigma}^{\dagger} c_{j, \sigma}+\text { h.c. }\right) \\
& +U \sum_{i} n_{i, \uparrow} n_{i, \downarrow}
\end{aligned}
$$

where $n_{i}=c_{i, \sigma}^{\dagger} c_{i, \sigma}$ for $\sigma=\uparrow$ and $\downarrow ; c_{i, \sigma}$ is an annihilation operator of an electron at the $i$ th site with spin $\sigma$; the sum of the first line is taken over the nearest neighbor pairs $\langle i, j\rangle$ in a single layer of the nanographite; the sum of the second line is taken over sites where the distance between two positions of the neighboring layers is shortest; $t_{1}$ is the strength of the weak hopping interaction between neighboring layers; the positions of $t_{1}$ are shown by the filled circles in Fig. 1 (a) (nanographite a) and Fig. 1 (c) (nanographite c); the interaction $t_{1}$ is considered at all the sites in Fig. 1 (b) (nanographite b); and the last term of the hamiltonian is the strong onsite repulsion with the strength $U$.

The finite size system is diagonalized numerically, and we obtain two kinds of solutions. One of them is a nonmagnetic solution where up and down spin electrons are not polarized in each layer. This kind of solutions can be found in weak $U$ cases. The other kind of solutions is an antiferromagnetic solution, where the number of up spin electrons is larger than that of down spin electrons in the first layer, the number of down spin electrons is larger than that of the up spin electrons in the second layer, and so on. This kind of solution is realized in strong $U$ regions. There will be cases of incommensurate spin density waves, but we have not obtained 
such kinds of solutions by choosing initial magnetic ordered states, which are commensurate with the one dimensional lattice in the stacking direction, at the first stage of the numerical iteration process. The present author has discussed the antiferromagnetism in $\mathrm{C}_{60}$ polymers [8]. The same technique used in Ref. [8] is effective in this paper, too.

The parameters are changed within $0 \leq t_{1} \leq 0.5 t$ and $0 \leq U \leq 10 t$. The realistic value of $t_{1}$ is estimated to be about $0.1 t$ at most, but we change this parameter for more extended regions in order to look at the behaviors of solutions in detail. All of the quantities of the energy dimension are reported using the unit $t(\sim 2.0-3.0 \mathrm{eV})$.

\section{Magnetic moment per layer}

First, we consider the total magnetic moment per layer for the nanographites a and $\mathbf{b}$. Figure 2 shows the absolute value of the total magnetic moment per layer as functions of $t_{1}$ and $U$. Figures 2 (a) and (b) are for the nanographite a, and Fig. 2 (c) is for the nanographite b. See the figure caption for the value of $U$ of each plot. Figure 2 (a) shows the overall variations of the magnetic moment. When $U$ is small, there appears a finite magnetic moment for the values of $t_{1}$ larger than the threshold of the phase transition. At $U=2.5 t$, the magnetic moment changes like a function of a parabola with respect to $t_{1}$. The magnetic moment decreases for larger $U: U=3.0 t, 5.0 t$, and 10.0t. This is due to the strong singlet correlation at the bonds $t_{1}$ with respect to the change of the Heisenberg coupling between the neighboring layers as $t_{1}^{2} / U$. Figure 2 (b) shows the details around the phase transition for $1.8 t \leq U \leq 2.3 t$. As increasing $U$, the magnitude of the magnetization increases. The magnetic moment is zero at the smaller $t_{1}$ region for $U=1.8 t, 1.9 t, 2.0 t$, and $2.1 t$. The magnetic moment is zero only at $t_{1}=0$ for $U=2.2 t$ and 2.3t. We can understand the parabolic curves as a change due to the Heisenberg coupling proportional to $t_{1}^{2} / U$. The antiferromagnetic solutions really exist for larger $U$ regions in the A-B stacking case. On the other hand, Fig. 2 (c) shows the magnetic moment for the simple stacking. There is not magnetization for $0 \leq t_{1} \leq 0.5 t$ and $0 \leq U \leq 10 t$. This 
is a remarkable difference between the simple A-A and A-B stackings, and is a new finding of this paper. There is not any clear evidence which stacking is realized experimentally [3]. However, we believe that the A-B stacking should exist in nanographite systems because the exotic magnetisms have been observed in recent experiments [1-3]. The increase of the magnetic interaction while attachment of water molecules [3] is induced by the decrease of the interlayer distance, which enhances the interaction strength $t_{1}$.

Next, Fig. 3 shows the absolute value of the magnetic moment per layer for the nanographite c with A-B stackings. The carbon number in a layer is 54, and is more than twice as large as that of the nanographite a. Figure 3 (a) shows the change of the magnetic moment for wide parameter regions, and Fig. 3 (b) displays the numerical data around the phase transition. The overall behaviors in the smaller and larger $U$ seem similar to those of Fig. 2. The characteristic value of $U$ decreases from that of Fig. 2. For example, the curve becomes parabolic for $U \geq 2.2 t$ in Fig. 2 (b), and it becomes parabolic for $U \geq 2.0 t$ in Fig. 3 (b). Such the quantitative difference is due to the effects of the larger system size.

\section{Local magnetic polarization in a layer}

In order to give further insights into mechanism of the magnetism, we will look at the local magnetic moments which depend on the carbon sites in each layer. We particularly pay attention to the local magnetism near the edge sites of the nanographite [4-6].

Figure 4 shows the local magnetic moment at the edge sites of the nanographite a. The values of $U$ are $U=2.1 t$ and 2.5t in Figs. 4 (a) and (b), respectively. In the former case, there is a point of the phase transition near $t_{1}=0.25 t$, and there appears a finite magnetization for nonzero $t_{1}$ in the latter case. The filled squares, open squares, and filled circles show the results at sites A, B, and C, respectively. Due to the symmetry, the magnetic moments at the sites A', B', and C' of Fig. 1 (a) are equal to those of the sites A, B, and C. Figure 5 displays the similar

plots for the nanographite c. The parameters are $U=1.8 t$ and $2.5 t$ in Figs. 5 (a) and (b), 
respectively. We see that the local magnetic moment is negative along the edge A-A' in Fig. 1 (a), and also along the edge A-B-A' in Fig. 1 (c). The local magnetic moment is positive along the neighboring edges: namely, the edges B-C and B'-C' in Fig. 1 (a), and the edges C-D-E and C'-D'-E' in Fig. 1 (c). Such the positive and negative alternations of magnetic moments are seen in both calculations of the nanographites a and $\mathbf{c}$. Because there are strong amplitudes of wavefunctions at the zigzag edge sites [4-6], the local moments near these edge carbon atoms tend to become larger. In the A-B stackings, there is not an interlayer interaction $t_{1}$ at the edge sites, and this gives rise to the finite magnetic moment per layer which has been discussed in the previous section. On the other hand, the local magnetic moment and also the magnetic moment per layer do not appear in the simple A-A stacking case namely the nanographite $\mathbf{b}$ of Fig. 1 (b), owing to the interlayer interactions $t_{1}$ which are present between all the nearest carbon atoms of neighboring layers. This difference is the origin of the fact that there is not magnetization in the simple stacking case reported in Fig. 2 (c).

In the band calculations of the stacked nanographite ribbons [9], the strong hybridization between edge states occurs in the A-A stacking case. Such the hybridization is weak in the A-B stacking case. The strong localization of wavefunctions at the edge carbon sites persists in the band calculations for systems with the A-B stacking [9], and this property agrees with the present result.

\section{Density of states}

In this section, we discuss the local density of states at the edge sites. The wavefunctions of electrons with up and down spins are projected on the edge sites of the nanographite a and $\mathbf{c}$. The local density of states is reported together with the total density of states.

Figure 6 shows the density of states of the nanographite a, and Fig. 7 displays the result of the nanographite c. The total density of states per layer and per spin is shown by the bold line. The local density of states at the edge sites is shown by the thin and dashed lines for the 
up and down spins, respectively. The up and down splitting typical to the antiferromagnetism is seen in both figures. Because the number of edge sites is one third of that of the total carbon atoms in the nanographite $\mathbf{a}$, the areas between the lines and the horizontal axis have such the relative ratios. In the nanographite $\mathbf{c}$, the portion of the edge sites with respect to the total carbon number becomes smaller. Therefore, the relative area below the thin and dashed lines becomes smaller in Fig. 7. In one dimensional graphite ribbons [4-6], there appears a strong peak due to the localized edge states at the Fermi energy. This is seen in the non-interacting case. With interactions taken into account, such the edge states split into bonding (occupied) and antibonding (unoccupied) states. This fact will be one of the reasons why such the strong peak is not observed in Figs. 6 and 7. Also, in the present case, the edge sites do not make a one dimensional lattice and each layer has a finite spatial dimension. Such the difference will be the second reason of the absence of the strong peak.

\section{Summary}

In summary, we have theoretically investigated the stacking effects in the zigzag nanographite sheets. We have found a remarkable difference in the magnetic properties between the simple A-A and A-B type stackings. For the simple stacking, there are not magnetic solutions. For the A-B stacking, we find antiferromagnetic solutions for strong onsite repulsions. The local magnetic moments exist at the edge sites due to the large amplitude of wavefunctions at the zigzag edge sites. The A-B type stacking is favorable in order that the exotic magnetism is observed in nanographite systems.

\section{Acknowledgements}

The author is grateful for interesting discussion with T. Enoki, T. Kawatsu, T. Ohshima, Y. Miyamoto, K. Kusakabe, K. Nakada, K. Wakabayashi, and M. Igami. Useful discussion with the members of Condensed Matter Theory Group (http://www.etl.go.jp/ ${ }^{\sim}$ theory/), Electrotechnical Laboratory is acknowledged, too. 


\section{References}

[1] Y. Shibayama, H. Sato, T. Enoki, and M. Endo, Phys. Rev. Lett. 84, 1744 (2000).

[2] N. Kobayashi, T. Enoki, C. Ishii, K. Kaneko, and M. Endo, J. Chem. Phys. 109, 1983 (1998).

[3] N. Kawatsu, H. Sato, T. Enoki, M. Endo, R. Kobori, S. Maruyama, and K. Kaneko, Meeting Abstracts of the Physical Society of Japan 55 Issue 1, 717 (2000).

[4] M. Fujita, K. Wakabayashi, K. Nakada, and K. Kusakabe, J. Phys. Soc. Jpn. 65, 1920 (1996).

[5] M. Fujita, M. Igami, and K. Nakada, J. Phys. Soc. Jpn. 66, 1864 (1997).

[6] K. Nakada, M. Fujita, G. Dresselhaus, and M. S. Dresselhaus, Phys. Rev. B 54, 17954 (1996).

[7] R. Saito, M. Yagi, T. Kimura, G. Dresselhaus, and M. S. Dresselhaus, J. Phys. Chem. Solids 60, 715 (1999).

[8] K. Harigaya, Phys. Rev. B 53, R4197 (1996).

[9] Y. Miyamoto, K. Nakada, and M. Fujita, Phys. Rev. B 59, 9858 (1999). 


\section{Figure Captions}

Fig. 1. Stacked nanographite with zigzag edges. The bold and thin lines show the first and second layers, respectively. The stacking is the A-B type in (a) (nanographite a) and (c) (nanographite $\mathbf{c}$ ), and it is the simple A-A type in (b) (nanographite b). There are 24 carbon atoms in one layer in (a) and (b), and there are 54 atoms in one layer in (c). The filled circles in (a) and (c) show lattice positions with small interlayer interactions $t_{1}$, and the bold symbols indicate some of edge sites in the first layer. The sites, A (B, C ...) and A' (B', C' ...), are symmetrically equivalent, respectively.

Fig. 2. The magnitude of the total magnetic moment per layer as functions of $t_{1}$ and $U$. Figures (a) and (b) are for the nanographite a, and Fig. (c) is for the nanographite b. In (a), the values of $U$ are $U=1.5 t$ (filled squares), 2.0t (open squares), 2.5t (filled circles), 3.0t (open circles), $5.0 t$ (filled triangles), and 10.0t (open triangles), respectively. Figure (b) shows the details around the phase transition: the values of $U$ are $U=1.8 t$ (filled squares), $1.9 t$ (open squares), $2.0 t$ (filled circles), $2.1 t$ (open circles), $2.2 t$ (filled triangles), and 2.3t (open triangles), respectively. In (c), the magnetic moment is zero for $0 \leq U \leq 10 t$.

Fig. 3. The magnitude of the total magnetic moment per layer as functions of $t_{1}$ and $U$ for the nanographite c. In (a), the values of $U$ are $U=1.0 t$ (filled squares), $1.5 t$ (open squares), $2.0 t$ (filled circles), $2.5 t$ (open circles), $5.0 t$ (filled triangles), and 7.0t (open triangles), respectively. Figure (b) shows the details around the phase transition: the values of $U$ are $U=1.2 t$ (filled squares), 1.4t (open squares), 1.6t (filled circles), $1.8 t$ (open circles), $2.0 t$ (filled triangles), and $2.2 t$ (open triangles), respectively.

Fig. 4. Local magnetic moment at the edge sites of the nanographite a. The values of $U$ are $U=2.1 t$ in (a), and 2.5t in (b). The filled squares, open squares, and filled circles show the 
results at sites $\mathrm{A}, \mathrm{B}$, and $\mathrm{C}$, respectively.

Fig. 5. Local magnetic moment at the edge sites of the nanographite c. The values of $U$ are $U=1.8 t$ in (a), and $2.5 t$ in (b). The filled squares, open squares, filled circles, open circles, and filled triangles show the results at sites A, B, C, D, and E, respectively.

Fig. 6. Density of states per layer of the nanographite a. The parameters are $t_{1}=0.4 t$ and $U=2.1 t$. The bold line shows the density of states over 24 carbon atoms per layer and per spin. The thin and dashed lines indicate the density of states over the eight edge sites in a layer for the up and down spins, respectively.

Fig. 7. Density of states per layer of the nanographite c. The parameters are $t_{1}=0.4 t$ and $U=1.8 t$. The bold line shows the density of states over 54 carbon atoms per layer and per spin. The thin and dashed lines indicate the density of states over the twelve edge sites in a layer for the up and down spins, respectively. 
(a)

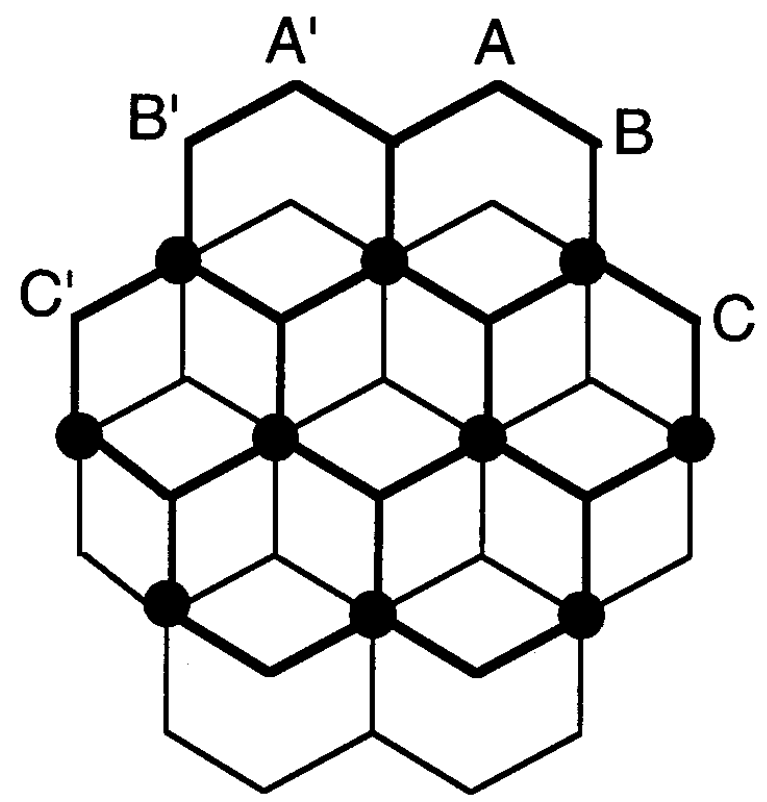

(b)

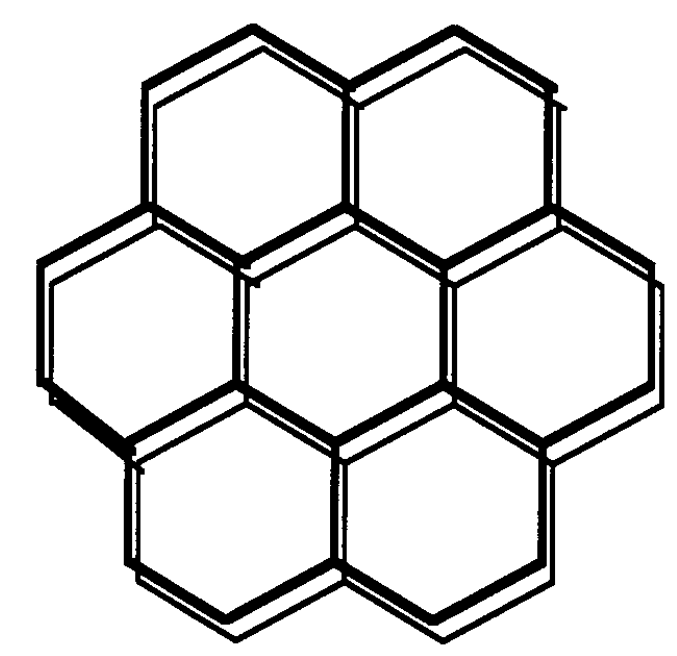


(c)

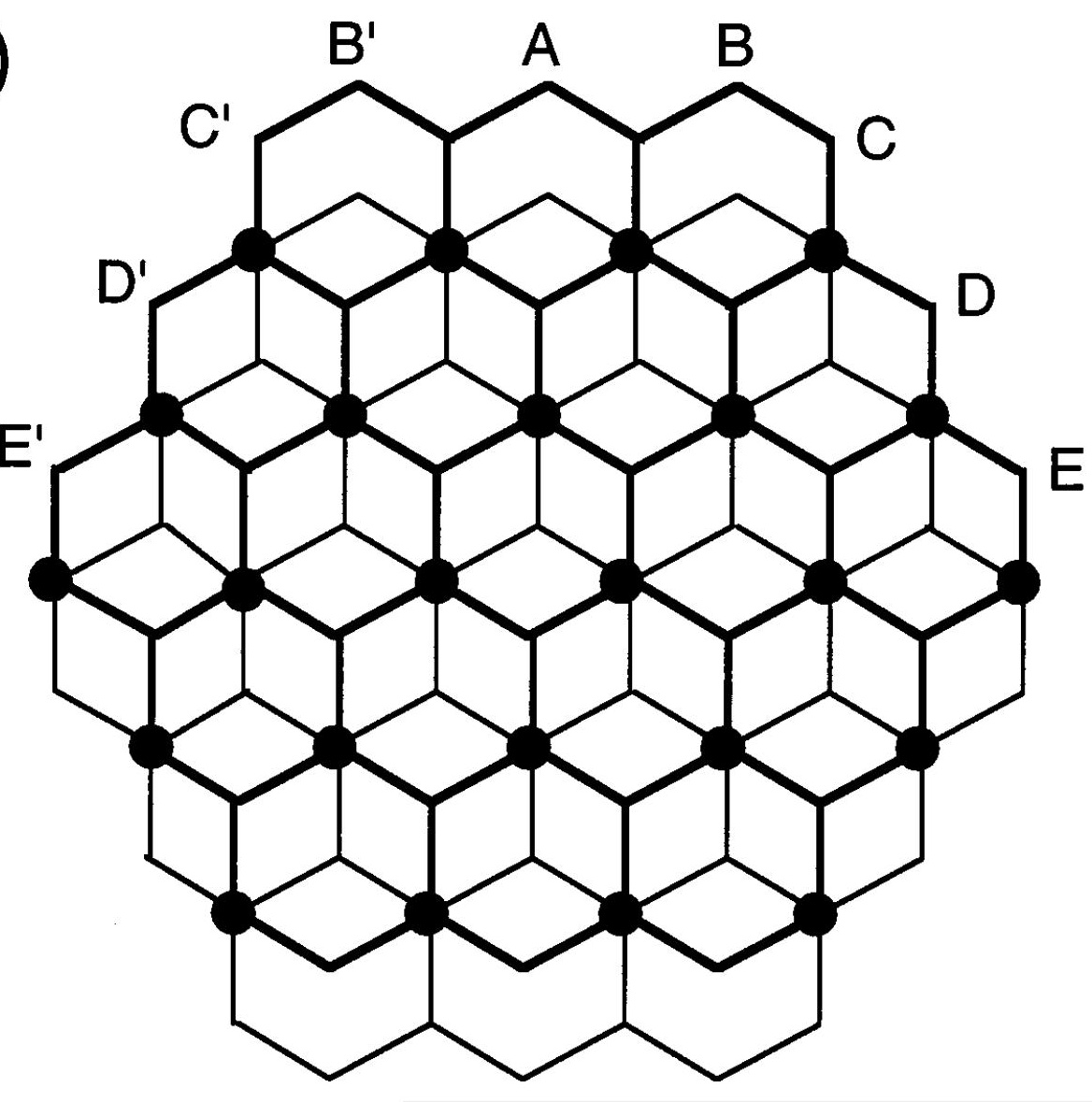



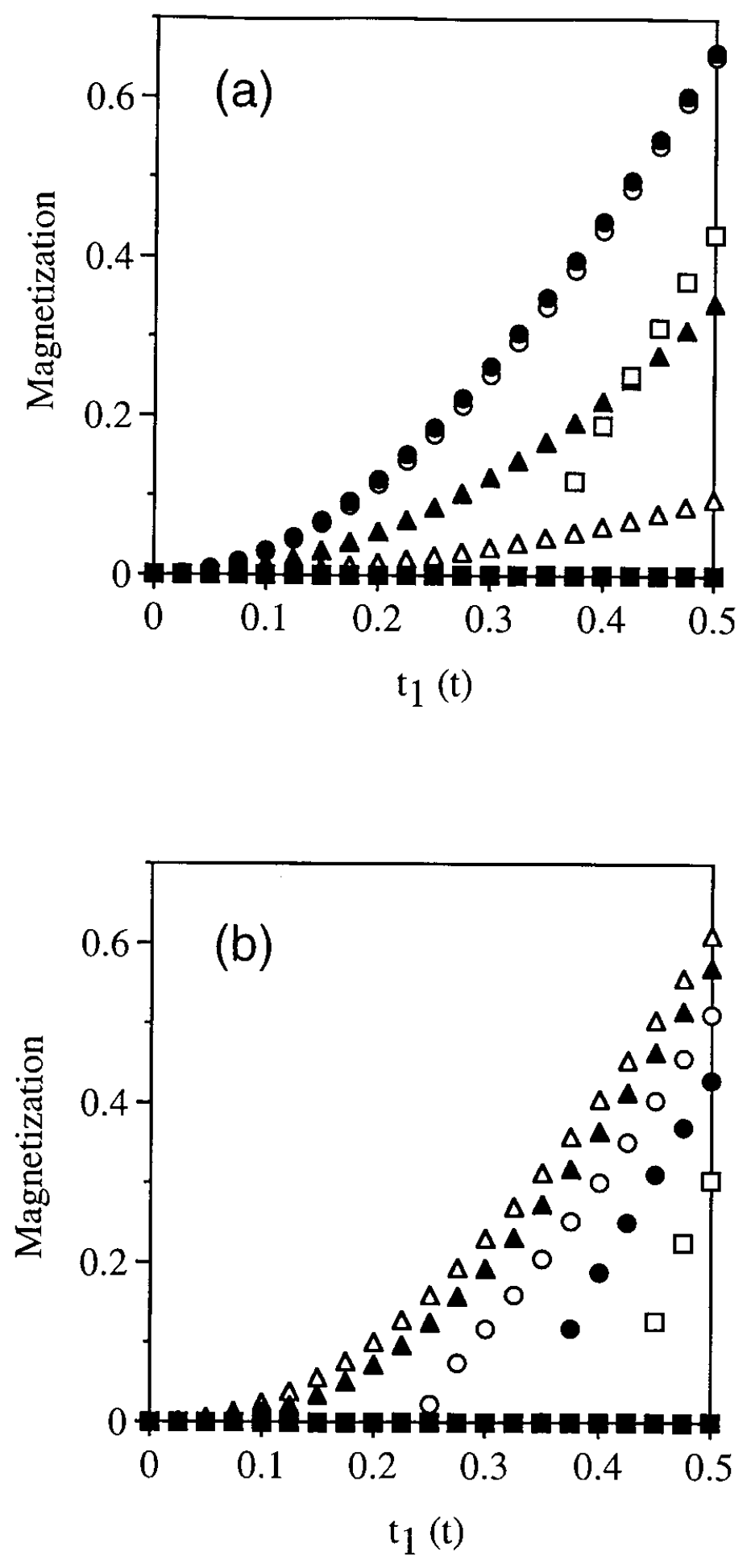


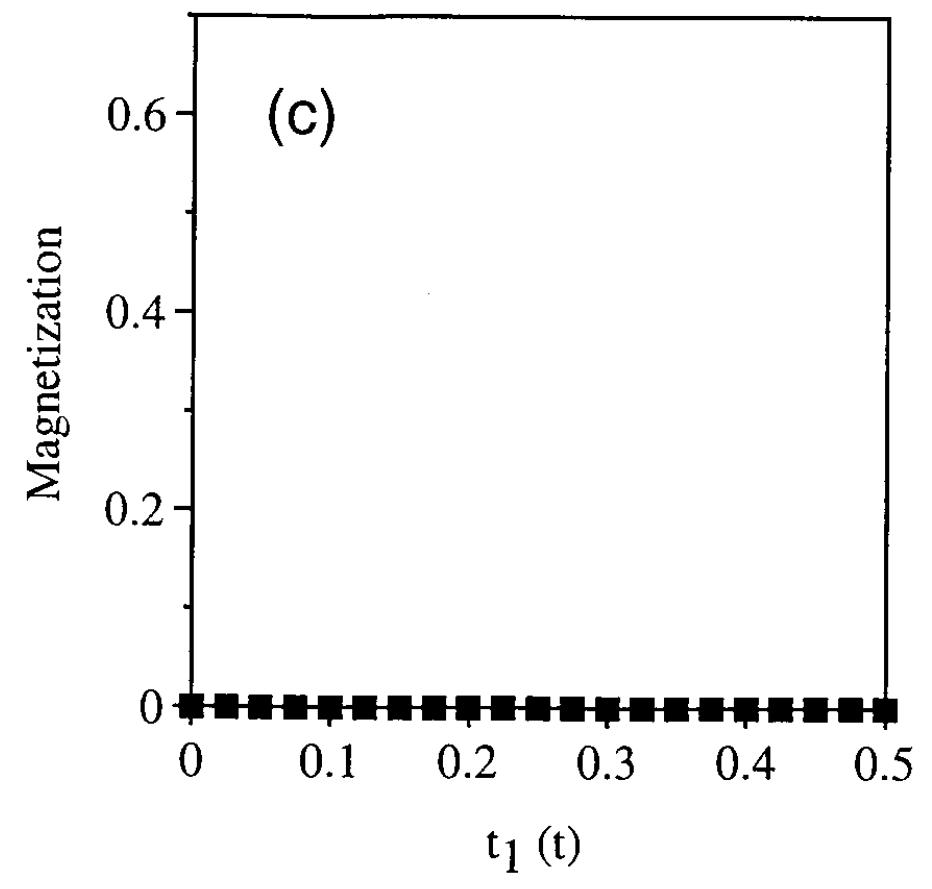



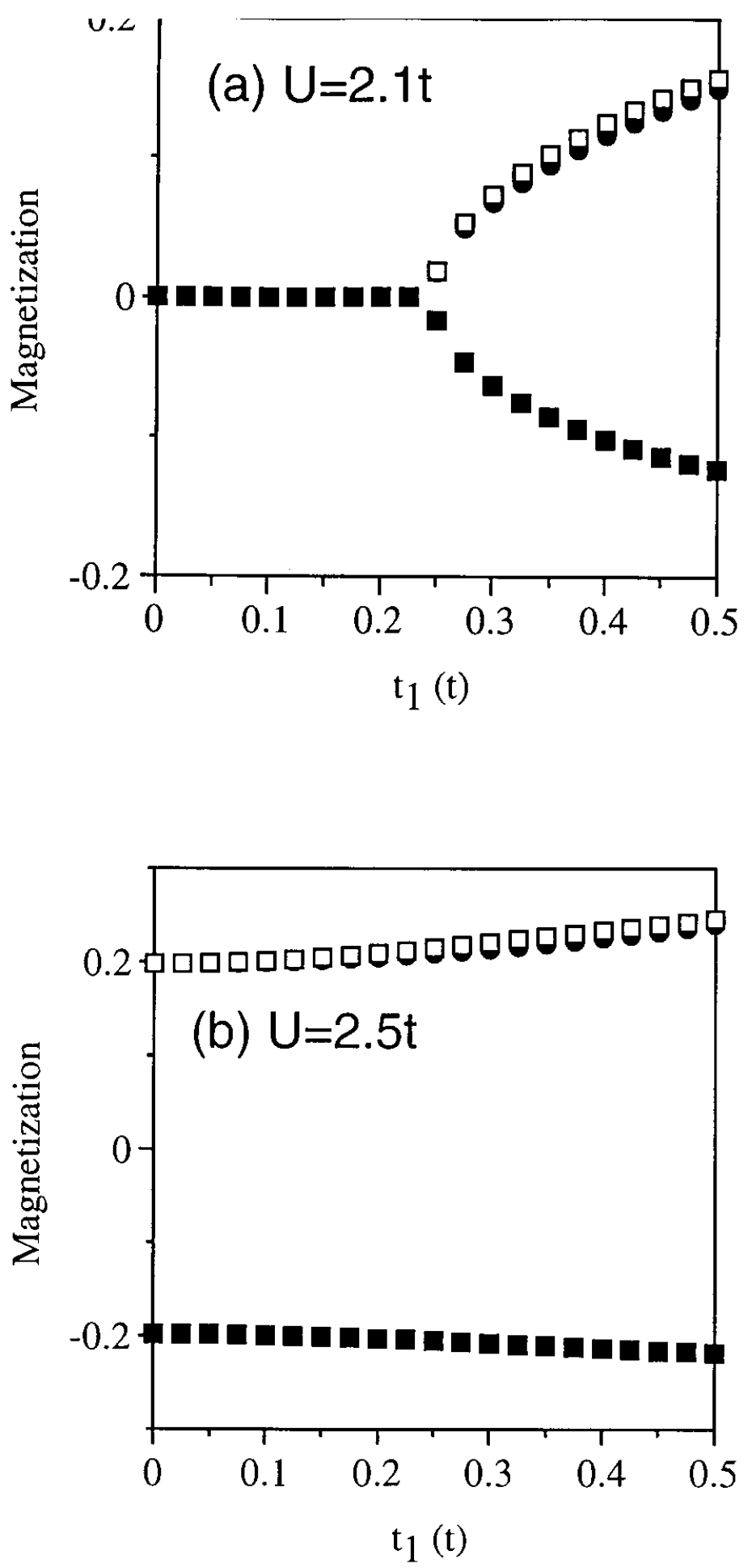

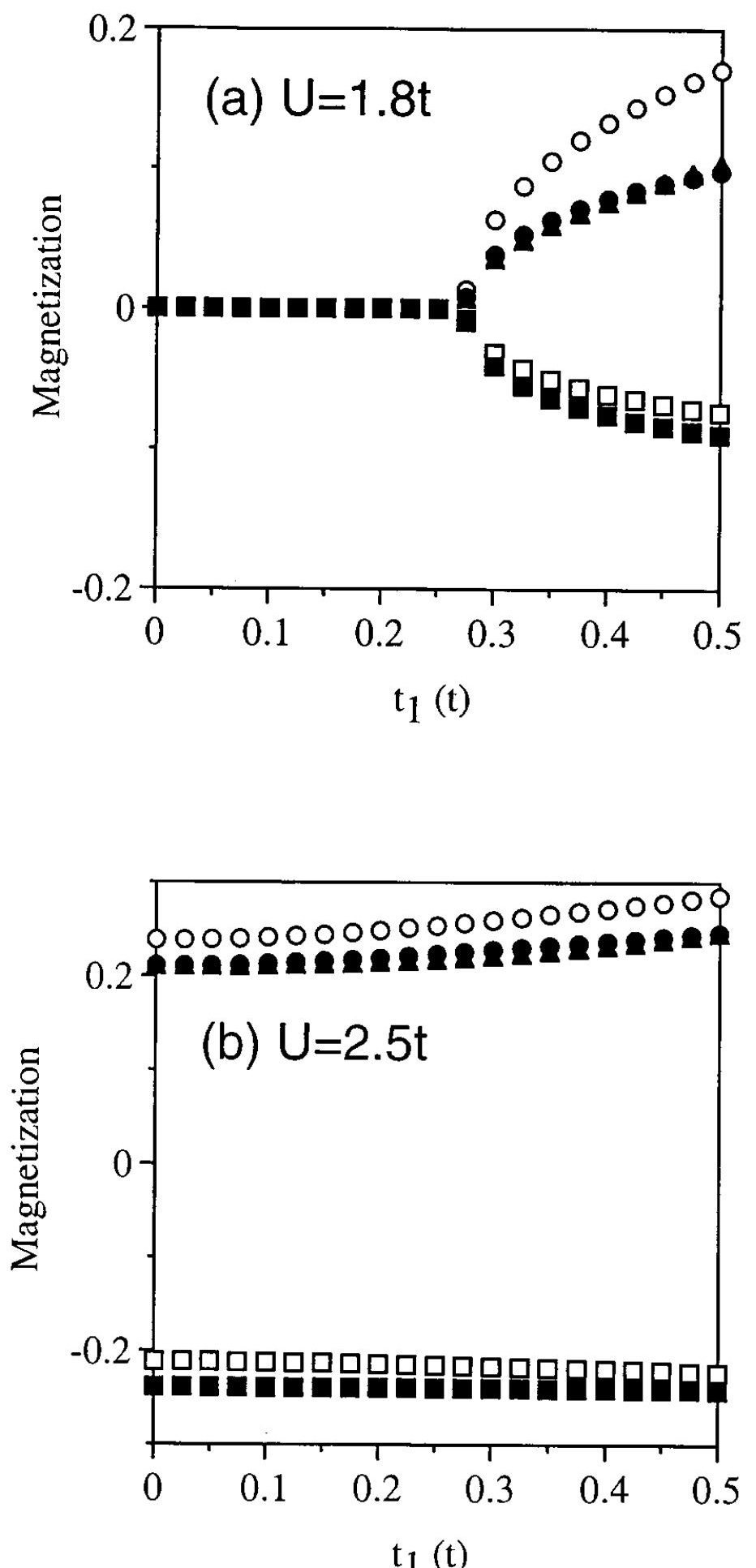


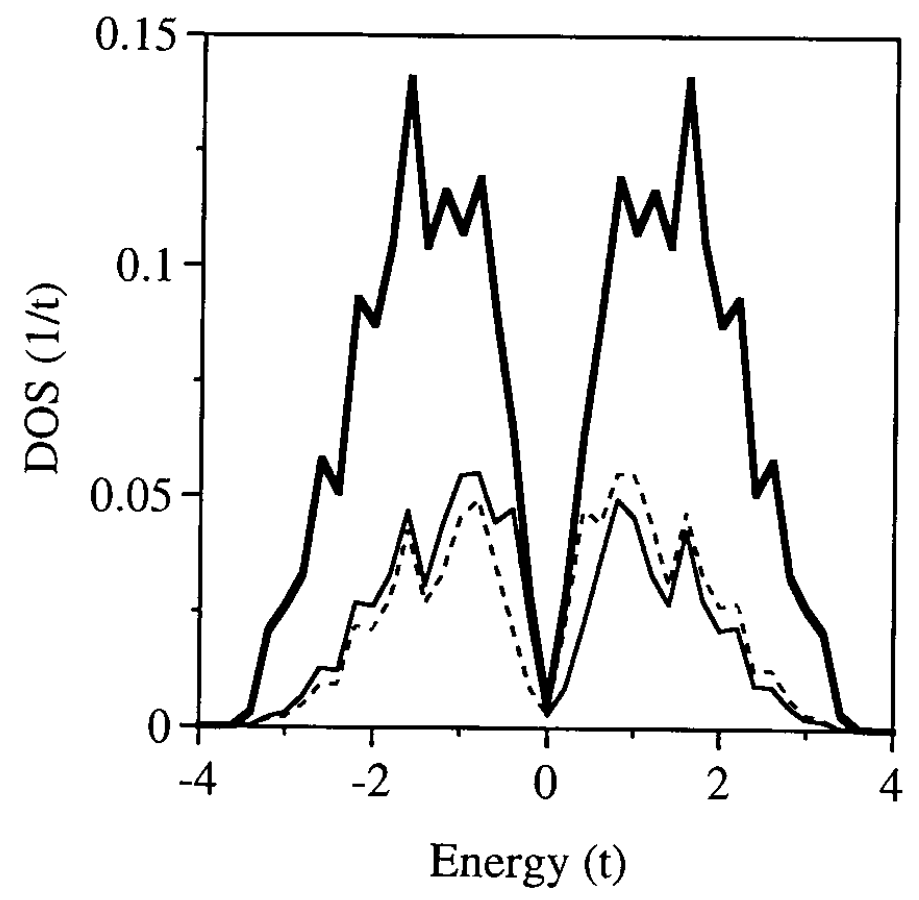




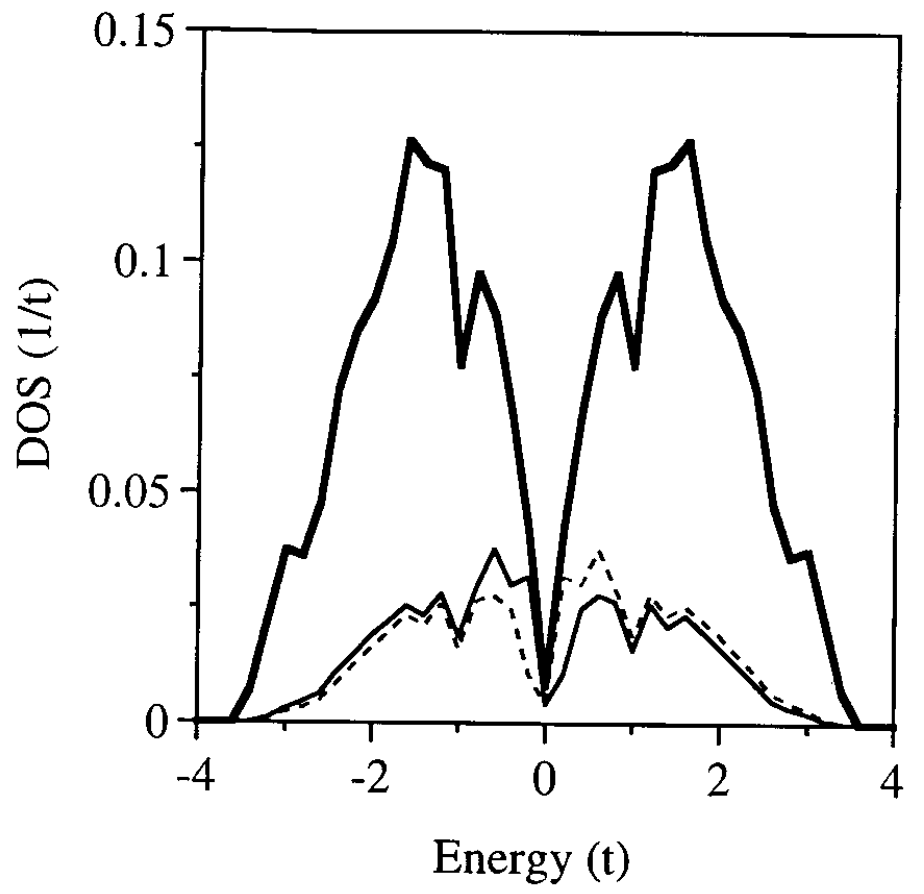

